\title{
Economía de los datos y propiedad
} sobre los datos*

Data economy and data ownership

\author{
$M^{a}$ Carmen Plana Arnaldos \\ Profesora Titular de Derecho Civil \\ Universidad de Murcia \\ E-mail: mcarmenp@um.es
}

\begin{abstract}
Resumen: La denominada revolución digital y la economía de los datos han traído consigo un debate social y doctrinal acerca de cómo se debe articular el marco legal aplicable para adaptarlo a las características y necesidades que plantea la digitalización. En este contexto, la cuestión de a quien pertenecen los datos, la propiedad sobre los datos, adquiere una especial importancia, ya que de facto circulan en el mercado generando grandes beneficios. En este trabajo se analizan las diferentes posturas doctrinales acerca de la propiedad sobre los datos, los obstáculos que se plantean para el reconocimiento de tal categoría, así como algunas posibles alternativas de regulación.
\end{abstract}

Palabras clave: economía de los datos, propiedad, libre circulación de los datos, propiedad sobre los datos.

\footnotetext{
* Trabajo realizado en el seno del proyecto de investigación "El Derecho de contratos en el entorno digital", financiado por el Ministerio de Ciencia, Innovación y Universidades (PGC2018-098206-B-I00).
} 


\begin{abstract}
Technological revolution and data economy have triggered an academic and social discussion about how the legal framework should be adapted to the specific characteristics and problems of the digitization. In this context, the question of who owns data, data ownership, is particularly important because data are de facto exchanged in commerce and produce large benefits. This paper analyses different scholar opinions about data ownership, mismatches of this category, and explore some legal alternatives.
\end{abstract}

Keywords: data economy, ownership, free flow of data, data ownership.

Sumario: 1. Planteamiento. 2. El proceso de obtención de rendimiento económico de los datos: raw data y datos elaborados. 3. El debate social sobre la participación de los sujetos en los beneficios económicos generados por los datos. 4. Propiedad sobre los datos. 4.1. Propuestas doctrinales que sirven de base a los movimientos sociales que defienden la existencia de un derecho de propiedad sobre los datos. 4.2. Distinción entre el derecho sobre los datos personales y otros datos. 4.3. La posible base jurídica en los derechos de contenido patrimonial reconocidos en el RGPD. 4.4. Los datos personales como contraprestación contractual en la Directiva (UE) 2019/770 y en el TRLGDCU. 4.5. Indisponibilidad de los datos personales. 4.6. Acceso a la información y libre circulación de los datos. 4.7. El control sobre los datos no necesita el reconocimiento de un derecho de propiedad. 5. Propiedad sobre los datos no personales. 6. Conclusiones. 7. Bibliografía.

\title{
1. Planteamiento
}

La denominada revolución digital, que se extiende desde el ámbito tecnológico, al económico y al social, ha cambiado sustancialmente la sociedad actual y es un desafío para el Derecho y los juristas, que tienen que responder a las necesidades puestas de manifiesto por nuevas formas de relación surgidas en el entorno digital (DE FRANCESCHI, A. - SCHULZE, R., 2019). Como sabemos, la economía digital se basa en tecnologías de la información que han convertido los datos en el centro de la actividad económica, ya que son la materia prima en la que se basa y los que generan grandes beneficios. La economía digital es, realmente, una economía de los datos. El rápido desarrollo de innovaciones como el análisis de datos, la fabricación automatizada, la gestión basada en análisis de probabilidad y otras muchas, está generando un valor global inusitado de la información digital. 
La economía de los datos mide la repercusión global del mercado de los datos - es decir, el mercado en que se intercambian datos digitales como productos o servicios derivados de los datos brutosen el conjunto de la economía. Implica la generación, recogida, almacenamiento, procesamiento, distribución, análisis, elaboración, entrega y explotación de los datos que hacen posibles las tecnologías digitales (European Data Market Study, SMART 2013/0063, IDC, 2016).

En este contexto, es especialmente importante el debate jurídico en torno a la atribución de derechos al particular sobre los datos que genera, tanto personales como de otro tipo. Y es que, según se ha puesto de manifiesto, el desarrollo de la llamada economía de los datos se ha producido sin derecho de propiedad, los datos son una especie de res communis ómnium generada por nuestra actuación en la red, que son utilizados por las grandes corporaciones como activos (commodities) de los que obtienen grandes beneficios económicos. La cuestión de la atribución de un derecho de propiedad sobre los datos se ha planteado tanto a nivel social y político, como a nivel doctrinal.

\section{El proceso de obtención de rendimiento económico de los datos: raw data y datos} elaborados

El desarrollo tecnológico, en especial el big data, ha creado una economía basada en los datos en la que intervienen diferentes sujetos, integrados en un proceso complejo que tiene como resultado una mejora económica basada en la innovación. El proceso de mercantilización de los datos se desarrolla de forma compleja a través de una cadena que comienza en los sujetos que producen o aportan datos; éstos son recogidos, sistematizados y analizados por empresas que tienen capacidad para ello; y por último se extrae su valor económico, que en muchas ocasiones genera innovación (ZECH, H., 2017, 2-3).

Se pueden obtener los datos de los sujetos de diferentes formas: en primer lugar, con el consentimiento expreso de un sujeto, normalmente en el marco de una relación contractual, para obtener determinados bienes o servicios, en los casos en los que los datos constituyen la contraprestación contractual (PLANA ARNALDOS, 2020). Además, en la relación con el proveedor del servicio se van generando datos que también recibe el prestador, os recopila, sistematiza, analiza y utiliza para diferentes fines, y, en ocasiones, cede a terceros, bien para prestar o mejorar el servicio, bien para otros fines lucrativos. En segundo lugar, no solo la cesión consciente y consentida es fuente de información para las empresas; cualquier actividad de los sujetos en el ámbito digital deja un rastro en forma de datos que puede ser utilizado por las empresas que operan en 
internet y que tienen la capacidad y la tecnología suficientes para procesar la información. Estos operadores utilizan la información como si se tratara de una res nullius apropiable por cualquiera (PERETE RAMIREZ, C. - GARCÍA MEXIA, P., 2020, 1684).

Los raw data (datos crudos o datos originarios) es decir, los datos que provienen directamente de los sujetos que los generan, ya sean facilitados por ellos mismos o fruto de su actividad, así como los datos que provienen de los dispositivos conectados (smart devices) son agregados, organizados y analizados a través de técnicas de big data, para finalmente inferir la valiosa información que se pretende. Se van sucediendo eslabones de una cadena en cada uno de los cuales los datos van adquiriendo valor económico. En esa cadena es necesaria una labor cualificada y una moderna y sofisticada tecnología para llegar a obtener fruto patrimonial de la materia prima constituida por esos raw data. No es un proceso sencillo, ni está al alcance de cualquiera; el valor económico de los datos solo se obtiene como fruto de la actividad de procesamiento de grandes cantidades de datos originarios que se convierten en datos procesados, que las empresas utilizan para distintos fines, como mejorar sus productos o servicios, optimizar costes, hacer ofertas o diseñar productos o servicios personalizados etc. Es realmente la tecnología del big data la que produce el valor económico de los datos; es común en la doctrina enumerar las características del big data con las "cuatro Vs": volumen, variedad, velocidad y veracidad, que describen perfectamente las condiciones que deben darse para obtener todo el valor económico que pueden proporcionar los datos. Así la tecnología del big data se basa en el manejo de grandes cantidades de datos, se antepone la cantidad a la calidad, ya que la idea es que el análisis de grandes cantidades de datos permite obtener una información nueva y cualitativamente muy útil (ZECH, H., 2017, 3). La variedad significa que las técnicas de big data pueden manejar diversos tipos de datos, no solo una cierta categoría. La velocidad hace referencia al tratamiento o procesamiento de datos en tiempo real. Y, por último, la veracidad se refiere a que los datos que se procesan sean ciertos, respondan a la realidad (excluye esta característica PRAINSACK, B., 2019, 1).

Esta mínima descripción del funcionamiento de la economía de los datos, que casi todos conocemos, al menos intuitivamente, nos sirve para ver la necesidad de diferenciar los derechos que se pueden atribuir a cada sujeto sobre los datos que genera; más concretamente, debemos distinguir los derechos que se atribuyen sobre los datos originarios (raw data) y los que se atribuyen sobre los datos elaborados. Mientras que en 
el primer caso el sujeto es claro, quien genera los datos, en el segundo caso debemos tener en cuenta la intervención de distintos sujetos en el proceso, y en especial la inversión, material y de trabajo, que quien elabora los datos ha hecho.

Pero no es solo la anterior la distinción que debemos tener en cuenta, también se debe plantear la diferencia entre los datos personales, los datos no personales, y los datos generados por maquinas. En cada caso, las alternativas jurídicas para la atribución de derechos son diferentes y también lo son las dificultades que podemos encontrar.

\section{El debate social sobre la participación de los sujetos en los beneficios económicos generados por los datos}

Diversos movimientos sociales en todo el mundo han planteado la cuestión de quien debe beneficiarse del potencial rendimiento de los datos personales (que parece incluir, en general, datos generados por el usuario). El ejemplo más claro lo encontramos en el movimiento francés Génération Libre que con el revelador slogan "Mes datas sont à moi" proclama el reconocimiento de un derecho de propiedad del titular sobre sus datos con el argumento de que los ciudadanos deben participar en los ingresos económicos generados por la explotación de sus datos personales. Siguiendo esta línea, el partido del gobierno francés La République en marche presentó una propuesta de ley en la Assemblée Nationale que sin embargo fue rechazada ${ }^{1}$. En Alemania, la coalición entre partidos políticos que formó el gobierno en 2017 proponía la propiedad de los datos en su contrato de coalición y en marzo de 2018 la canciller alemana Angela Merkel citó expresamente entre los objetivos del gobierno alemán la regulación de un sistema justo de propiedad de los datos que garantizara la participación de los particulares en su rendimiento económico ${ }^{2}$. En Estados Unidos, el candidato demócrata Andrew Yang incorporó a su programa electoral el reconocimiento de un derecho de propiedad de cada individuo sobre sus datos y, como facultades asociadas, el derecho a ser informado sobre la recopilación

\footnotetext{
${ }^{1}$ Projet de loi relatif à la protection des données personnelles. Texte adopté $\mathrm{n}^{\circ} 113$, session ordinaire du 15 mai 2018. Accesible en: http://www.assemblee-nationale.fr/15/ta/tap0113.pdf. Última consulta, mayo 2021. Última consulta julio 2021.

${ }^{2}$ Ein neuer Aufbruch für Europa-Eine neue Dynamik für Deutschland-Ein neuer Zusammenhalt für unser land, Koalitionsvertrag zwischen CDU, CSU und SPD, 19. Legislaturperiode (12 March 2018) 102. Accesible en: https://www.cdu.de/system/tdf/media/dokumente/koalitionsvertrag 2018.pdf?file=1. Última consulta, junio 2021.
} 
de los mismos y como se utilizarán, así como a obtener una parte del rendimiento generado por ellos.

Más allá de las declaraciones políticas, los llamados personal data services o personal data lockers permiten al titular de los datos gestionar su información personal, depositada en estos servicios, para que puedan controlarla de manera centralizada y utilizarla con la finalidad que decidan (no necesariamente patrimonial). Incluso algunas empresas proponen directamente que el titular obtenga una remuneración por el uso de los datos. Una sencilla búsqueda en internet nos muestra multitud de resultados de empresas que ofrecen la venta de "tus" datos personales (citizenme.com, Lotame.com, Dawex.com, wesellyourdata.com, Moneymagpie.com, etc.). En este mismo sentido, el Supervisor Europeo de Protección de Datos opinaba, en el año 2015, que las organizaciones que usen datos personales para tratamientos no relacionados con el servicio principal que prestan deben compartir la riqueza que genera la actividad con las personas cuyos datos han utilizado. El Supervisor llega incluso a proponer la creación de espacios personales en los que cada sujeto almacena información personal que puede generar rendimientos económicos ${ }^{3}$.

Todas estas iniciativas reflejan un creciente sentimiento social de que los datos personales y otros datos generados por los particulares crean un inmenso negocio en el que todos los actores se benefician económicamente, menos los particulares de los que provienen los datos $^{4}$. Aunque tal razonamiento puede resultar excesivamente simple, evidencia la necesidad de tener en cuenta la vertiente patrimonial de los datos generados por los usuarios, tanto los datos personales, como los generados por el sujeto en su actuación en internet y aquellos generados a través de los dispositivos conectados (smart devices) cada vez más frecuentes en nuestra vida diaria.

\footnotetext{
${ }^{3}$ Opinion 7/2015 del Supervisor Europeo de Protección de Datos "Meeting the challenges of Big Data. A Call for Transparency, User Control, Data Protection by Design and Accountability" 19 de noviembre de 2015. Accesible en https://edps.europa.eu/sites/edp/files/publication/15-11-19_big_data_en.pdf . Última consulta agosto 2021 .

${ }^{4}$ Vid. como ejemplo de debate social las referencias a los debates periodísticos organizados en EE.UU por "The Financial Times" (PURTOVA, 2015, 3).
} 
Probablemente, tal como se ha puesto de relieve en la doctrina (HUMMEL, P. - BRAUN, M. - DABROCK, P., 2020) el reconocimiento de un derecho de propiedad sobre los datos debería entenderse rectius como una demanda de redistribución de los beneficios generados por los datos, así como un reconocimiento social del valor de la generación de los mismos por los sujetos. No esta tan claro, sin embargo, que esta finalidad se pueda, o se deba, conseguir reconociendo un derecho de propiedad sobre los datos tal como lo conocemos en sentido tradicional.

\section{Propiedad sobre los datos}

La realidad económica y el debate social dejan claro que los datos, especialmente los datos personales, no pueden ser considerados jurídicamente solo desde el ámbito de la protección de la privacidad, o en sentido amplio, desde la perspectiva de protección de los derechos fundamentales (en especial el derecho a la protección de los datos personales y las normas que lo amparan). Teniendo en cuenta tales normas, y la especial naturaleza de los datos personales, es necesario abordar jurídicamente la vertiente patrimonial, la cesión, uso y explotación de los datos.

En tal sentido, existe alguna regulación aplicable en la Unión Europea (DUCH-BROWN, N. - MARTENS, B. - MUELLER-LANGER, F, 2017, 12-19): en relación con los datos personales, el RGPD y las normas que lo complementan, en España la LOPDyDD; en el caso de los datos generados por maquinas o datos industriales, entran en juego tanto las normas sobre secretos comerciales, como las normas que protegen las bases de datos. Junto a ellas, también existen algunas reglas contractuales, como las Directivas (UE) 2019/770 y 2019/771, que han sido transpuestas en España en el TRLGDCU. Todas estas normas permiten solucionar algunos problemas en ciertos supuestos, pero la cuestión objeto de debate radica en si es necesaria una regulación organizada y genérica de un derecho de propiedad sobre los datos que reconozca y discipline la capacidad de disposición de los sujetos sobre los mismos.

A nivel legislativo ya hemos visto algunos intentos de regular este derecho (RITTER, J. - MAYER, A., 2018, 228-239). Junto a la iniciativa francesa, también en EE.UU el reconocimiento de la propiedad sobre los datos está presente en el debate político y ha llegado al ámbito legislativo. En marzo de 2019, a iniciativa del senador republicano por 
Louisiana Kennedy, se presentó en el Senado un proyecto de Ley "para prohibir la recolección de datos o información en internet" (Own your own data Act. A Bill to prohibit the collection of data or information generated on the internet) ${ }^{5}$. Esta propuesta reconocía expresamente en su art. 2 que cada individuo tiene un derecho de propiedad exclusivo sobre sus datos generados en internet (un concepto muy amplio que incluye datos personales y otros generados por el usuario y dispositivos conectados). La California Consumer Privacy Act 2018 (CCPA) ${ }^{6}$ reconoce el derecho inalienable a la privacidad de todas las personas, que se traduce en la posibilidad del sujeto de controlar el uso de su información personal, incluida expresamente la venta; es decir, reconoce una vertiente patrimonial.

La doctrina privatista se ha ocupado extensamente de la cuestión de la mercantilización de los datos y los derechos que se deberían reconocer al sujeto del que provienen, pero las respuestas ofrecen muy diversas alternativas, que van desde la defensa del derecho de propiedad sobre los datos, hasta su negación, con base, ambas posturas contrapuestas, en distintos argumentos.

El recurso al concepto de propiedad sobre los datos para explicar jurídicamente el derecho del sujeto sobre sus datos tiene una gran fuerza, probablemente porque es una institución jurídica que todos conocemos. Cuando un sujeto se refiere a sus datos, reconduciéndolo al concepto de propiedad está incluyendo cualquier poder de decisión sobre ellos, tanto la protección de la privacidad, como el poder para compartirlos o no y el de obtener las ganancias que puedan derivar de su uso.

Si nos fijamos en los trabajos doctrinales, numerosísimos, que tienen por objeto la propiedad sobre los datos, podemos comprobar que en ellos se reflejan diferentes conceptos de propiedad (THOUVENIN, F. y TAMÓ-LARRIEUX, A., 2021, 317): en primer lugar, la propiedad se puede entender en su sentido estricto, como deriva de las normas del Código civil español (en nuestro caso) o bien como propiedad intelectual, ambos integran el concepto tradicional de propiedad; en estos casos, los argumentos que

\footnotetext{
${ }^{5}$ Accesible en https://www.govtrack.us/congress/bills/116/s806/text. Última consulta julio 2021.

${ }^{6}$ Accesible:http://leginfo.legislature.ca.gov/faces/codes_displayText.xhtml ?awCode=CIV\&division=3.\&t $\underline{i t l e=1.81 .5 . \& p a r t=4 . \& \text { chapter }=\& \text { article. }}$. Última consulta agosto 2021 .
} 
se utilizan pasan por asimilar los caracteres de los datos y el poder sobre ellos a los caracteres propios del derecho de propiedad o de propiedad intelectual. En segundo lugar, la propiedad sobre los datos se puede entender en sentido amplio como derecho que garantiza control sobre los datos, en especial pensando en los datos personales; de ahí que en estos casos los argumentos para defender la existencia de un derecho de control, concebido como exclusivo y por tanto calificado de propiedad, se busque en las normas que regulan el derecho a la protección de datos personales, en especial el RGPD.

En este sentido, lo fundamental es la garantía de un derecho de acceso del sujeto a los datos personales. Realmente, sobre la base de la denominación propiedad sobre los datos encontramos diferentes propuestas sobre la regulación patrimonial de los datos, articulando el poder del sujeto de control y aprovechamiento económico sobre los mismos.

En todo caso, aplicar el concepto (tradicional) de propiedad a los datos encuentra algunos obstáculos derivados de la peculiar naturaleza del objeto: el titular puede garantizar el acceso a otro sujeto (por ej. el proveedor del contenido en internet) pero no transmite la posesión, como es paradigmático en los bienes objeto de propiedad; no se garantiza un acceso exclusivo, cualquier otro sujeto puede tener acceso con consentimiento y al mismo tiempo, y el propio titular puede seguir usando los datos; el uso temporal es otro elemento distorsionador, ya que la transmisión fundada en un derecho de propiedad es definitiva, incluso en los casos en los que no implica pérdida total para el titular, el adquirente sí lo es de manera definitiva en el tiempo, lo que no ocurre en los datos. El valor de la información está en su intercambio a tiempo real, y se deprecia muy rápido, por lo que no parece compatibilizar con un derecho exclusivo con vocación de permanencia (DUCH-BROWN, N. - MARTENS, B. - MUELLER-LANGER, F., 2017, 14).

Probablemente el principal obstáculo para calificarlo como derecho de propiedad es que no se puede concebir como un derecho exclusivo, ya que el consentimiento para tratar datos no excluye su uso por el titular, ni impide la autorización a otros sujetos (DREXL, 2018, 63). 


\title{
4.1. Propuestas doctrinales que sirven de base a los movimientos sociales que defienden
} la existencia de un derecho de propiedad sobre los datos

Como venimos diciendo, la categoría jurídica de la propiedad, y el reconocimiento de un derecho de propiedad sobre los datos, es la herramienta jurídica utilizada por algunos movimientos europeos y norteamericanos (en este país, en especial el pionero de la realidad virtual, J. LANIER) tanto para explicar el poder de control del sujeto sobre sus datos, como, fundamentalmente, ordenar un sistema económico, la economía de los datos, permitiendo a todos los actores, también los sujetos de los que provienen los datos, participar en los beneficios económicos que se generan.

Así, el reconocimiento del derecho de propiedad sobre los datos, defendido en Francia por el movimiento Generation libre (think tank liberal), con base en la aportación doctrinal de algunos juristas (BINCTIN, V., 2018; LANDREAU, I. - PELIKS, G. BINCTIN, N. - PEZ-PÉRARD, V., 2018). es realmente la vía para obtener una redistribución que entienden más justa de los beneficios de los datos, que hasta ahora, sostienen, quedan en manos exclusivamente de grandes empresas capaces de tratar la información. Es por ello que las consideraciones en las que basan su propuesta son, además de jurídicas, éticas y filosóficas, convirtiendo el reconocimiento de la propiedad sobre los datos en una opción que refleja el concepto liberal de la sociedad y la economía que defienden. Los liberales, declaran, deben reclamar la propiedad privada y la remuneración, a diferencia de otros movimientos, como los socialistas que proponen impuestos y redistribución, o los social-demócratas, que proponen la vía del derecho de obligaciones. Resulta sumamente clara la declaración inicial del rapport base de esta teoría?:

\begin{abstract}
Aujourd'hui, nos données personnelles sont dans la nature, res nullius appropriées et revendues par les grands acteurs du numérique. Face à cette situation inique, chaque école de pensée tente d'imaginer une réponse : les sociaux-démocrates inventent des droits et obligations, les socialistes proposent des schémas de taxe et redistribution, les nationalistes imaginent une souveraineté numérique...Il est important que les libéraux fassent également entendre leur voix en plaidant pour la propriété privée et la rémunération. Proudhon considérait la propriété comme «la plus grande force révolutionnaire qui existe », en ce qu'elle confère à l'individu la souveraineté sur son domaine propre. Il n'y pas de maîtrise sans possession. Ce droit fondamental doit aujourd'hui s'étendre aux données, prélude d'une véritable propriété de soi sur soi.
\end{abstract}

\footnotetext{
${ }^{7}$ Accesible en https://www.generationlibre.eu/wp-content/uploads/2018/01/2018-01-generationlibrepatrimonialite-des-donnees.pdf . Última consulta agosto 2021.
} 
Las opciones jurídicas se ven determinadas por los planteamientos ideológicos. Los argumentos jurídicos utilizados por los autores del movimiento Generation Libre parten de la regulación del Code civil francés (BINCTIN, V., 2018, 56-62) pero pueden ser asumidos a la luz del Código civil español en su mayor parte. Son los siguientes: los datos se pueden incluir en el concepto de bienes, objeto de propiedad, como se incluyen los bienes inmateriales. Algunos defienden firmemente el reconocimiento de un derecho de propiedad sobre los datos y sus argumentos parten de considerar la información, una vez recogida (recorded), como un bien material, un tangible (RITTER, J. - MAYER, A., 2018, 220-277); pueden ser objeto de apropiación y control, caracteres esenciales de la propiedad; además son poseíbles, de la misma forma que se admite la posesión inmaterial; el poder de hecho estaría representado por la posibilidad de mantener los datos en secreto. Por ello, se compara con la regulación de los secretos comerciales, sobre los cuales se admite la posibilidad de licencias.

En consecuencia, el régimen de la propiedad, incluida su defensa frente a cualquiera, es aplicable a toda clase de datos, personales y no personales. Conforme a este planteamiento también se reconoce un derecho de propiedad a las empresas que obtiene los datos. Para evitar esta conclusión la doctrina en la que se basa este movimiento entiende que el RGPD impide la apropiación de los datos personales (LANDREAU I., 2018, 77, 83, 85-87) y por ello propone la regulación específica del régimen de la propiedad sobre los datos personales.

Frente a los razonamientos expuestos, DREXL, J. (2019, 32-33) ha puesto de manifiesto que la base jurídico-normativa de esta teoría se centra exclusivamente en el derecho francés, lo que supone que las soluciones no son exportables a otras legislaciones, y no sirve de nada una regulación nacional de un derecho de propiedad sobre los datos, que funcionan en un mercado digital global. De otro lado, al citado autor le resulta llamativo que no se presente un estudio económico para una propuesta que tiene como base la existencia de un mercado de datos no equilibrado, en el que las grandes empresas se benefician del rendimiento económico que generan los datos, mientras que los sujetos de los que provienen son meros "durmientes" (fournisseurs endormis); pero, sobre todo, que no se tenga en cuenta el efecto que la propiedad sobre los datos puede tener en el funcionamiento del mercado y respecto al principio de libre circulación de los datos, que está en la base de desarrollo económico en la sociedad actual. Y es que la protección del 
interés público hace que la regla general deba ser el libre acceso a los datos, y solo justificadamente se podrán reconocer derechos exclusivos y/o excluyentes sobre los mismos (DREXL, 2018, 3, 23 ss, 33-34).

En Alemania defiende un derecho de propiedad intelectual sobre los datos no personales KERBER, W. (2016). FEZER, K-H. (2017) proponía un derecho de propiedad sobre los datos personales amparado por las normas de protección de datos (RGPD) y sobre los datos generados por el usuario y dispositivos conectados, concebido como un nuevo derecho de propiedad intelectual que tendría como base jurídica directa la Constitución alemana. Esta teoría merece para DREXL, J. $(2019,33)$ la misma critica que la anterior. Con todo, la construcción propuesta ha sido matizada por el autor en un trabajo más reciente para la Konrad Adenauer Foundation (think tank del partido político CDU) en el que propone una propiedad representativa sobre los datos, caracterizada por la participación colectiva en los beneficios, dada la dificultad de articular la remuneración a cada sujeto en particular, creando una agencia gubernamental que se encargue de ingresar y distribuir los beneficios (FEZER, K-H., 2018) .

Las dos propuestas doctrinales referidas, que son las que mayor difusión han tenido en el debate sobre los derechos de los sujetos sobre los datos en Europa, constituyen, como se ha señalado, la base jurídica de las propuestas políticas que han tenido lugar en los países europeos en los que han surgido (provienen de los think tank de los partidos de gobierno de sus países). Resulta evidente la relación entre las propuestas jurídicas, las propuestas políticas y el sentimiento social. La fórmula para articular jurídicamente los derechos de los sujetos sobre los datos a través del reconocimiento del derecho de propiedad plantea múltiples desajustes y puede ser discutible, pero no podemos olvidar que el Derecho debe dar respuesta a las demandas y necesidades sociales y, por tanto, como ya hemos señalado, es necesario articular normas que regulen la vertiente patrimonial de los datos. En este sentido, DREXL, J. $(2019,21)$ entiende que hay dos alternativas: regular el ámbito patrimonial de los datos a través de una regulación moderna ( $a$ modern commercial data law) que se aplique de manera uniforme a todos los datos, tanto personales como de otro tipo (tengamos en cuenta que en muchas ocasiones es

\footnotetext{
${ }^{8}$ Una propuesta parecida en su sentido último la podemos encontrar en PURTOVA $(2015,29)$ que llega a la "conclusión intuitiva" de una propiedad colectiva sobre los datos personales de pequeños grupos o comunidades, alternativa a la propiedad individual.
} 
sumamente difícil distinguir entre datos personales y los que no lo son entre los datos producidos por los sujetos, sobre todo en los datos de navegación y los producidos por dispositivos o maquinas). O bien, otra solución consistiría en una regulación separada de los datos personales y otro tipo de datos. En esta segunda opción, la primera de estas regulaciones estaría limitada y seguiría los principios de las normas relativas al derecho fundamental a la protección de datos personales, una legislación protectora de los sujetos y que contemple los datos como bienes de la personalidad; mientras que la segunda estaría presidida por el principio de creación de un mercado de datos eficiente. Con todo, incluso en esta segunda opción es necesario tener en cuenta que los datos no pueden ser tratados como una simple mercancía, tal como ha puesto de relieve el Supervisor Europeo de Protección de Datos, en su conocido Informe 4/2017 sobre la Propuesta de Directiva (UE) 2019/770, sobre contenidos digitales ${ }^{9}$.

El autor citado propone una legislación única que tenga en cuenta ambos aspectos señalados, la protección de los aspectos ligados a la personalidad de los sujetos, y la regulación de un mercado eficiente en que se respete el principio de libre circulación de los datos, en aras de la protección de los intereses generales (DREXL, J., 2018, 3, 23). Como destaca el informe de OSBORNE CLARKE $(2016,100)$ el reconocimiento de un derecho de propiedad sobre los datos depende de sus efectos económicos. Los aspectos patrimonial y personal están unidos y la regulación debe tener en cuenta ambos.

\subsection{Distinción entre el derecho sobre los datos personales y otros datos}

El principal argumento que justifica buscar un mecanismo de control por el sujeto sobre los datos, articulándolo jurídicamente como propiedad, es el vinculo entre el sujeto y sus datos personales. Se justifica un derecho de propiedad sobre los datos sobre la base de que están indisolublemente ligados a la persona y por tanto son suyos, razonamiento intuitivo que solo cabe respecto a los datos personales. Probablemente este es el motivo por el que los mayores esfuerzos doctrinales tienen por objeto la justificación de los derechos patrimoniales de los sujetos sobre los datos de carácter personal (PURTOVA, N., 2015, 4).

\footnotetext{
${ }^{9}$ Accesible en https://edps.europa.eu/sites/edp. Última consulta agosto 2021.
} 
A este primer argumento podemos añadir que los datos personales tienen probablemente un valor patrimonial per se mientras que otro tipo de datos adquieren su valor como resultado de su agregación y tratamiento a través de técnicas de big data. Así, los derechos sobre los datos originarios y los derechos sobre los datos elaborados no serían del mismo sujeto ya que, para obtener los segundos, las empresas han de disponer de una tecnología, utilizar medios materiales y humanos altamente especializados $y$, en suma, invertir para obtener el rendimiento. La actividad necesaria para obtener el rendimiento de los datos elaborados ha llevado a algunos autores (FEZER, K-H., 2017) a preconizar un derecho de propiedad intelectual nuevo o característico, distinto de la propiedad tradicional en cuanto a la categoría, que los sujetos tendrían sobre los datos, basado precisamente en la actividad y esfuerzo, material e intelectual, que se ha desarrollado para obtenerlo. Más allá del diseño jurídico que se defienda, lo cierto es que tal inversión no se realizaría si a quien la hace se le priva del rendimiento económico que produce, aunque otra cosa es la posible medida de participación en tal beneficio de los distintos sujetos que participan en el proceso: los sujetos de los que provienen los raw data y las empresas que producen los datos elaborados. En cualquier caso, parece clara la necesaria diferenciación entre los derechos sobre los datos personales y otros datos.

\subsection{La posible base jurídica en los derechos de contenido patrimonial reconocidos en el $R G P D$}

El reconocimiento de ciertos derechos del titular en el marco de la protección de datos personales (en la línea de hacer efectivo el repetido empoderamiento del titular de los datos) se ha querido ver como un reconocimiento de la existencia de un derecho de explotación patrimonial (VICTOR, J.M, 2013). El RGPD garantiza una serie de derechos que reflejan el control que se le reconoce al titular de los datos. Por un lado, el consentimiento es la legitimación principal del tratamiento, el sujeto decide sobre si se pueden tratar o no sus datos, al menos como regla general. Y resulta aún más claro el poder de control en la garantía de la libre revocación del consentimiento y el derecho de supresión de los datos (derecho al olvido). La cuestión es si estos poderes de control pueden entenderse como reflejo de la existencia de un derecho exclusivo y excluyente del titular sobre los datos, un derecho de propiedad. Debemos partir de que los derechos contenidos en el RGPD tienen como finalidad la garantía de un derecho fundamental y tienden a protegerlo, de ahí que en el Cdo 1 RGPD se conecte con el art. 8 de la Carta 
Europea de Derechos Fundamentales, que se refiere a la protección de la privacidad, y no con el art. 17, referido a la propiedad. Se trata de poderes ligados a la protección de la propia persona, no poderes de contenido patrimonial, ni pueden ser entendidos como reflejo de un derecho patrimonial como la propiedad. El marco jurídico de protección de datos personales es una herramienta para conseguir la protección de los derechos de la personalidad (privacidad entendida en sentido amplio) y hay que interpretar las reglas establecidas conforme a esta finalidad, incluso aquellas previsiones, como el reconocimiento del derecho a la portabilidad de los datos (art. 20 RGPD) que es claramente un derecho económico, pero se conecta más bien con el derecho de acceso, y no es reflejo de un derecho patrimonial de disposición sobre los datos personales. La portabilidad hay que interpretarla en clave económica, pero de mercado, garantizar la libre competencia, pero no como manifestación de un poder patrimonial exclusivo y excluyente del titular, como sería la propiedad (GONZÁLEZ PACANOWSKA, I., 2020). Así pues, el RGPD no puede ser interpretado como base para la defensa de un derecho patrimonial exclusivo del titular, porque esa no es la finalidad que pretende. Esta norma opera en el plano del derecho fundamental de protección de datos, y reconoce derechos en éste ámbito. Cuando el titular celebra un contrato por el que consiente el uso de sus datos personales, no está transmitiendo la propiedad de sus datos personales; ese consentimiento, aunque tenga una motivación económica, no convierte a la otra parte en propietario de los datos personales (PLANA ARNALDOS, $\mathbf{M}^{\mathrm{a}}$. C., 2020).

\subsection{Los datos personales como contraprestación contractual en la Directiva (UE)} 2019/770 y en el TRLGDCU

El art. 3 de la Directiva (UE) 2019/770 incluye en el ámbito de aplicación de la misma los contratos de suministro de contenidos o servicios digitales en los que el consumidor (párrafo 2) "[...] facilite o se comprometa a facilitar datos personales al empresario, salvo cuando los datos personales facilitados por el consumidor sean tratados exclusivamente por el empresario con el fin de suministrar los contenidos o servicios digitales con arreglo a la presente Directiva o para permitir que el empresario cumpla los requisitos legales a los que está sujeto, y el empresario no trate esos datos para ningún otro fin”. Se iguala, en los contratos entre empresarios y consumidores, precio en dinero y datos personales a efectos de considerar la existencia de un contrato sinalagmático. En consecuencia, se aplica a estos contratos, en los que el consumidor facilita o se compromete a facilitar 
datos personales, el régimen contractual previsto en la Directiva, especialmente el régimen de falta de conformidad.

Debemos llamar la atención sobre dos puntos que resultan importantes a los efectos que tratamos. En primer lugar, el precepto mencionado no se refiere a la transmisión de los datos, sino que utiliza un término (facilitar) que más bien podemos entender como una cesión. Por otro lado, se elude el término contraprestación (PLANA ARNALDOS, M C., $^{\mathrm{a}}$. 2020) probablemente para cumplir las rotundas exigencias del Supervisor Europeo de Protección de Datos, que en su Dictamen 4/2017 sobre la Propuesta de directiva era contrario a la utilización del término, ya que los datos no se pueden considerar una mercancía. En todo caso, la opción finalmente utilizada evita que con base en la Directiva los datos personales puedan ser considerados bienes apropiables. La finalidad de la Directiva es proporcionar un régimen uniforme de protección al consumidor tanto en los casos en los que paga un contenido o servicio en dinero, como cuando recibe el contenido o servicio aparentemente gratis, pero realmente a cambio de sus datos. El tratamiento uniforme se limita a la protección en caso de falta de conformidad y los remedios ante el incumplimiento. El sencillo razonamiento según el cual "si los puedo facilitar es que son míos" no parece suficiente para fundar en las previsiones de la Directiva el reconocimiento de un derecho de propiedad sobre los datos personales, sobre todo teniendo en cuenta la finalidad de esta previsión normativa.

La Directiva (UE) 2019/770, junto a la Directiva (UE) 2019/771, sobre contratos de compraventa de bienes, han sido transpuestas al ordenamiento jurídico interno español mediante el RDL 7/2021 de 27 de abril, que incluye nuevas normas en el TRLGDCU. La transposición española recoge expresamente que los datos personales pueden ser la contraprestación que el consumidor facilita al suministrador, a cambio de acceder a los contenidos y servicios digitales (CÁMARA LAPUENTE, S., 2021, 32-37). El nuevo artículo 59.4 TRLGDCU deja claro, como ya podíamos afirmar conforme a la Directiva, que en los supuestos en los que el usuario facilita datos personales a cambio de un contenido o servicio digital existe un contrato sinalagmático, en el que los datos personales son la contraprestación. Pero de la regulación contenida en los nuevos preceptos del TRLGDCU no podemos en modo alguno deducir que se transmita la propiedad o un derecho exclusivo del usuario sobre los datos personales, sino solo la autorización para el tratamiento y uso de los datos por el prestador del servicio o 
proveedor de contenido (CÁMARA LAPUENTE, S., 2021, 34); y es que, en este punto, la transposición sigue fielmente lo establecido en la Directiva, con la única modificación del término que se utiliza (contraprestación) que no deja lugar a dudas sobre la naturaleza contractual del intercambio.

\footnotetext{
Desde un punto de vista puramente terminológico, la utilización de la expresión en castellano "contraprestación" probablemente no presenta los mismos problemas que sugería el SEPD en sus consideraciones, ya que se trata de un término más amplio que no se usa solo en sentido puramente patrimonial, a diferencia del término inglés (counter-performance) que se evita precisamente para dejar claro que los datos personales no tienen un contenido patrimonial, al menos no exclusivamente (PLANA ARNALDOS, $\mathrm{M}^{\mathrm{a}}$. C., 2020, 586).
}

En suma, ni los derechos garantizados en el RGPD, ni el reconocimiento de la posibilidad de celebrar contratos a cambio de datos personales, que se consideran contratos sinalagmáticos a efectos de aplicar las normas contractuales, pueden ser considerados argumentos suficientes para entender que estamos ante el reconocimiento legal de un derecho de propiedad sobre los datos personales. Con todo, la declaración normativa del carácter contractual de estos supuestos supone el reconocimiento de una vertiente patrimonial en el uso de los datos, y no solo eso, sino la necesidad de que se regule.

La regulación de la economía digital, y por tanto de los derechos patrimoniales sobre los datos, debería tener como base, siguiendo las observaciones de DREXL, J. (2019, 21) los siguientes principios, que están en línea con las directrices marcadas por la Unión Europea: establecer un mercado funcional y competitivo, promover la innovación, proteger a los consumidores, con especial incidencia en la protección de la privacidad de las personas físicas y, por último, proteger otros intereses públicos que requieren la libre circulación de la información (seguridad, salud pública, investigación, etc).

\subsection{Indisponibilidad de los datos personales}

Frente al reconocimiento de un derecho de propiedad sobre los datos personales se esgrime su carácter indisponible. Los datos personales, protegidos por un derecho fundamental y reflejo de los derechos de la personalidad (intimidad, honor) son por naturaleza indisponibles, lo que no es compatible con el reconocimiento de un derecho patrimonial sobre ellos como la propiedad. 
Matizando este razonamiento, se ha señalado que la indisponibilidad no es un obstáculo insalvable, ya que es posible distinguir entre la titularidad en si misma, que no se perdería y no se puede transmitir, y ciertas facultades, como la de uso, que se puede transmitir a terceros (NAVAS NAVARRO, S., 2018, 106). Esta concepción ya es conocida en ciertos derechos de la personalidad, como la imagen, a los que se les reconoce un aspecto patrimonial, o la propiedad intelectual, en la que se diferencia el derecho moral, intransmisible, y el aspecto patrimonial, que puede ser objeto de explotación económica. Resulta evidente que los datos, también los personales, se transmiten en el mercado por motivos económicos y por ello realmente no es posible asumir una indisponibilidad absoluta. Con base en esta última afirmación, la doctrina se ha esforzado en intentar aunar, con diversas construcciones, la indisponibilidad natural de los datos personales y la realidad de su comercio. PURTOVA, N. $(2015,7)$ pone de relieve que, partiendo del valor económico de los datos, considerar y tratar los datos como res nullius, como parecen prima facie, no es realista. JANECEK, V. - MALGIERI, G. (2020) defienden lo que denominan una «alienabilidad dinámica» (dinamically limited alienability rule) en virtud de la cual los datos no se pueden considerar en si mismos transmisibles o intransmisibles, sino que deben considerarse intransmisibles cuando llevan consigo valores e intereses que se verían lesionados por la transmisión. Considerar los datos res extra comercio debe entenderse desde un punto de vista dinámico y no absoluto, teniendo en cuenta que la finalidad que persigue tal regla es proteger determinados valores.

En suma, la realidad se impone y es necesario reconocer la patrimonialidad tanto de los datos no personales, como la de los datos personales, al menos limitada (bien una vertiente patrimonial de los datos personales, o bien excluyendo algunos datos que consideramos sensibles o especialmente ligados a la personalidad).

\subsection{Acceso a la información y libre circulación de los datos}

El reconocimiento de un derecho de propiedad sobre los datos encuentra numerosos obstáculos, como la doctrina europea ha puesto de manifiesto y se ha tratado de exponer, pero probablemente la mayor objeción que se puede hacer tiene que ver con el interés público en el desarrollo de la economía de los datos y, para ello, en la libre circulación de los datos. El interés general determina que la regla deba ser el libre acceso a los datos, y solo justificadamente se podrán reconocer derechos exclusivos y/o excluyentes sobre los 
mismos. Esta regla tiene como fundamento la protección de la libertad constitucional de información y el interés público de acceso a los datos. Las limitaciones estarían representadas por las normas de propiedad intelectual y propiedad industrial, justificadas por el incentivo al desarrollo intelectual y tecnológico; y las normas de protección de datos personales, cuya restricción al tráfico de datos se justifica por la garantía de la privacidad y el derecho fundamental reconocido en las Constituciones europeas y en la Carta Europea de Derechos Fundamentales (DREXL, J., 2018, 23 ss., 33-34) ${ }^{10}$.

La UE viene declarando repetidamente desde 2015 que la economía digital y su desarrollo son básicos. La Estrategia Europea para un Mercado Único Digital"11 reconoce que la economía digital puede ampliar mercados y promover mejores servicios a mejores precios, ofrecer mayores posibilidades de elección y crear nuevas fuentes de empleo. Según declara la Comisión en su Comunicación de febrero de 2020: Una Estrategia Europea de Datos ${ }^{12}$, Europa aspira a aprovechar los beneficios de una mejor utilización de los datos, en particular una mayor productividad y unos mercados competitivos, así como mejoras en la salud y el bienestar, el medio ambiente, una gobernanza transparente y unos servicios públicos adecuados. Uno de los pilares fundamentales de la estrategia para un mercado único digital es la libre circulación de los datos dentro de la Unión, como garantía del desarrollo económico basado en la tecnología digital y los datos. La finalidad es asegurar que tanto los particulares como las empresas tiene acceso a los servicios y productos online en condiciones que garanticen la libre competencia, así como la protección de los consumidores y los derechos de propiedad intelectual.

Ahora bien, la libre circulación de los datos entendida de manera ilimitada resulta incompatible con el respeto de los derechos individuales, especialmente la privacidad y el derecho a la protección de datos personales. La necesidad de tener en cuenta ambos aspectos beneficia desde una perspectiva del interés general, reafirmando los valores de la Unión, y a la postre, desde una perspectiva económica, ya que la confianza de los consumidores es fundamental para conseguir el desarrollo de un mercado digital fuerte y

\footnotetext{
${ }^{10}$ PURTOVA, N. (2015,5) considera sin embargo que la dicotomía entre "propiedad privada" y datos personales como "bien público" no refleja la realidad, en la que de facto existe la propiedad sobre los datos personales.
}

${ }^{11} \mathrm{COM}(2015) 192$ final

${ }^{12} \mathrm{COM}(2020) 66$ final 
competitivo. Desde este punto de vista, la regulación patrimonial de los derechos de los particulares en relación a los datos, como por ejemplo las normas contractuales de protección del consumidor (así el regimen de falta de conformidad de la Directiva (UE) 2019/770) no responde solo a la defensa del interés particular de los consumidores, sino que al mismo tiempo se debe entender como un mecanismo de defensa de un interés patrimonial pero general; la defensa del consumidor pretende que éste permita, con base en la seguridad que la norma le proporciona, el libre tráfico de sus datos.

En definitiva, la regulación patrimonial de los datos, personales y de otro tipo, debe tener en cuenta la garantía de la libre circulación de los datos, aunando interés económico general y protección de los derechos de los particulares, y el reconocimiento de un derecho exclusivo y excluyente como es la propiedad no parece adecuarse a esta finalidad.

\subsection{El control sobre los datos no necesita el reconocimiento de un derecho de propiedad}

En los diversos y numerosos estudios doctrinales que tienen por objeto la propiedad sobre los datos podemos comprobar que subyacen dos concepciones diferentes de lo que se denomina propiedad de los datos; este concepto se entiende tanto como propiedad en sentido estricto, como también en el sentido de poder de control sobre los datos (THOUVENIN, F. - TAMÓ-LARRIEUX, A., 2021, 338). Aunque se usen en el mismo contexto, ambas concepciones tienen consecuencias diferentes. Por un lado, el concepto de propiedad tiene, como se ha visto, dos inconvenientes fundamentales: de un lado, incide negativamente en la libre circulación de los datos y puede ir por tanto en contra de intereses generales, tanto económicos como de otro tipo. Por otro lado, aplicar a los datos el concepto de propiedad plantea objeciones como el uso no exclusivo y excluyente, ya que los datos se pueden reproducir, no se priva al "transmitente" de su uso y pueden ser tratados por distintos sujetos al mismo tiempo (¿todos ellos poseedores? ¿todos ellos propietarios?). Aunque tales inconvenientes se pueden rebatir en parte, o minimizar su importancia, probablemente la finalidad que se pretende reconociendo la "propiedad sobre los datos" se puede obtener por otras vías jurídicas.

Se ha puesto de relieve, además, que el reconocimiento de este derecho no garantizaría de manera más efectiva el poder de control de los sujetos sobre los datos, sino al contrario, ya que es posible entender que las empresas los adquieren y por tanto, aplicando los 
principios de la propiedad, podrían excluir que el sujeto pueda seguir cediéndolos para otros fines o a otros sujetos. En esta línea, se sostiene por algunos autores que la vía adecuada para garantizar el control del sujeto sobre los datos personales son las normas y principios reconocidos en el RGPD, que establece mecanismos de control del sujeto sobre sus datos como el derecho de acceso, de cancelación y la portabilidad, que permiten un control efectivo de los datos personales (THOUVENIN, F. - TAMÓ-LARRIEUX, A., 2021, 338).

No debemos perder de vista, sin embargo, que el poder de control sobre los datos para ser efectivo desde el punto de vista patrimonial debe incluir no solo una vertiente defensiva a la que podría decirse que responden los principios y reglas del RGPD, sino que también es necesario regular la posición activa del sujeto en relación a sus datos; más claramente, lo que se debe plantear es la posibilidad del sujeto de obtener un beneficio directo de los datos a través de la cesión remunerada de su uso. Esta posibilidad es la que se ha puesto de manifiesto en los intentos legislativos de Francia y EE.UU, que se han mencionado anteriormente, y es probablemente lo que queda pendiente de regular y lo que se entiende, en suma, comprendido bajo el paraguas de la propiedad sobre los datos. En el caso de la regulación del derecho a la imagen, la posibilidad de obtener una remuneración por la cesión de uso está amparada y limitada sin necesidad de reconocer una propiedad sobre la imagen y, como se ha señalado, este podría ser el modelo para los datos personales.

\section{Propiedad sobre los datos no personales}

La cantidad de datos que se producirán, recogerán, almacenarán y analizarán en la economía digital irá en aumento. El desarrollo de la automatización de la producción, integrando máquinas y robots que producen e intercambian datos entre ellas, incluso a tiempo real, coches inteligentes y autónomos, o los dispositivos conectados (el llamado internet de las cosas) tienen como base la producción e intercambio de datos, que no son datos personales. Como pone de relieve KERBER, W. (2018) parte de estos datos no personales están protegidos a través de la propiedad intelectual (e-books, software, archivos digitales, etc.). Sin embargo, muchos de ellos, especialmente los generados por maquinas (machine-generated data and sensor-data) no se pueden entender incluidos en el ámbito de protección de estas normas, al menos tal como las conocemos actualmente. 
Conforme establece el Reglamento Europeo de libre circulación de datos no personales en su Cdo 9 " $[. .$.$] Entre los ejemplos específicos de datos no personales se encuentran los conjuntos de datos$ agregados y anonimizados utilizados para análisis de datos a gran escala, los datos sobre agricultura de precisión que pueden ayudar a controlar y optimizar la utilización de plaguicidas y de agua, o los datos sobre las necesidades de mantenimiento de máquinas industriales [...]" (Reglamento (UE) 2018/1807 del Parlamento Europeo y del consejo de 14 de noviembre de 2018 relativo a un marco para la libre circulación de datos no personales en la Unión Europea (DOUE L 303/59 de 28.11.2018).

Algunos autores han propuesto generalizar las reglas de la propiedad intelectual a todos los datos no personales, admitiendo una nueva forma de propiedad intelectual sobre los datos no personales (FEZER, K-H., 2017). Tal posibilidad resulta discutible, sobre todo porque los datos tienen una naturaleza industrial, están generados por maquinas y su funcionalidad se extrae cuando las maquinas interactúan entre si compartiendo la información (RITTER, J. - MAYER, A., 2018, 222) algo muy alejado de la creación intelectual y el fruto del ingenio humano que está en la base de la protección de la propiedad intelectual. Y por lo que se refiere a la protección del derecho sobre las bases de datos, el derecho de la UE (y el español) solo protege la base de datos, el conjunto de datos organizado ya existente, el resultado, pero no concede un derecho sobre los datos en si mismos ${ }^{13}$.

Como se ha señalado, cuando la generación de tales datos se produce mediante un proceso y a través de una infraestructura (de software y/o hardware) creados por una empresa, probablemente tal proceso y sus frutos, los datos, se podrían proteger por la vía de la propiedad intelectual, pero se trata de una posibilidad que habría que articular y que, en todo caso, habría de tener en cuenta que la protección de los derechos sobre los datos de quien invierte en conseguirlos y está justificado que tenga un retorno económico, se debe compatibilizar con el principio de libre circulación de los datos y el límite que impide el bloqueo de datos, porque de otro modo la garantía de funcionamiento de la economía de los datos, que, en el fondo justifica la protección, se vería perjudicada.

El debate sobre el reconocimiento de un derecho de propiedad sobre los datos no personales tiene como base la preocupación de las empresas por proteger el retorno económico de su inversión y, en definitiva, proteger el modelo de economía digital. No

\footnotetext{
13 OSBORNE CLARKE $(2017,7)$ en su informe pone de relieve que la decisión del TJUE en el caso Ryanair VS PR Aviation reconoce la libre regulación contractual relativa a los datos no protegidos en el ámbito de aplicación de las normas sobre bases de datos. De este modo, la vía contractual permitiría acuerdos que regularan aquellos aspectos necesitados de ello.
} 
es tanto la garantía de control de los datos, como ocurre en relación a los datos personales, sino más bien lo importante en este caso es potenciar el mercado y la libre circulación de los datos, por lo que es primordial la regulación del acceso a los datos y los principios en que se debe basar, no tanto la regulación del derecho de propiedad sobre los mismos. Algunos autores como DREXL, J. (2016 y 2018) no consideran necesario ni conveniente regular un nuevo derecho de propiedad sobre los datos no personales, precisamente porque influiría negativamente en la competencia y el mercado limitando el acceso público a la información ${ }^{14}$.

La Comisión Europea en su Comunicación: Hacia un espacio común europeo de datos ${ }^{15}$ se refiere al intercambio de datos entre empresas, que se produce: “en relación con datos que crean a menudo de forma automatizada máquinas y procesos basados en tecnologías emergentes como la Internet de las Cosas" y pone de relieve que consultadas las partes interesadas ${ }^{16}$, han puesto de manifiesto que no están a favor de la regulación de un derecho de propiedad sobre estos datos, destacando que la cuestión fundamental no es la propiedad sobre estos datos, sino la regulación del acceso a los mismos ${ }^{17}$. En este sentido THOUVENIN, F. - TAMÓ-LARRIEUX, A. (2021) optan por extender los derechos de control, en especial el derecho de acceso, contenidos en el RGPD a los datos no personales, lo que supondría alentar la economía de los datos.

El Reglamento Europeo para la libre circulación de datos no personales refleja estas consideraciones:

“[E]l funcionamiento eficaz y eficiente del tratamiento de datos es un componente fundamental en toda la cadena de valor de datos. No obstante, el funcionamiento eficaz y eficiente del tratamiento de datos y el desarrollo de la economía de los datos en la Unión se ven dificultados, en particular, por dos tipos de obstáculos a la movilidad de los datos y al mercado interior: los requisitos de localización de datos establecidos por las autoridades de los Estados miembros y las prácticas de

\footnotetext{
${ }^{14}$ Partiendo de un análisis económico también en contra del reconocimiento de un derecho de propiedad sobre los datos no personales generados por maquinas (industrial data) por ir en contra de la innovación y la competencia KERBER, W. (2018).

${ }^{15}$ COM (2018) 232 final

${ }^{16}$ Public Consultation on Building the European Data Economy. Accesible en https://eurlex.europa.eu/content/news/building_EU_data_economy.html. Última consulta agosto 2021.

${ }^{17}$ En este mismo sentido THOUVENIN, F. - TAMÓ-LARRIEUX, A. (2021) consideran que no hay razón para limitar a través de una regulación legal el libre tráfico de datos; al contrario, los datos desarrollarán todo su potencial si son compartidos entre empresas, por ejemplo, a través del reconocimiento de derechos de acceso.
} 
dependencia de un solo proveedor en el sector privado" (Cdo 2) "[L]a libre circulación de datos en la Unión va a desempeñar un papel importante para alcanzar un crecimiento y una innovación basados en datos" (Cdo 13).

Su finalidad principal es precisamente la garantía del principio que enuncia en su título y proteger el desarrollo de la economía de los datos, pero establece fundamentalmente obligaciones de los países para no entorpecer la libre circulación de los datos, dejando sin regular el ámbito de los derechos y obligaciones de las empresas y particulares en el tratamiento e intercambio de los datos no personales.

Podemos decir que existe un consenso doctrinal bastante extendido acerca de que no es necesaria la regulación de un derecho de propiedad sobre los datos industriales. Los autores destacan dos elementos: la garantía de la libre circulación y la posibilidad de regular los derechos sobre la recopilación de datos a través de un nuevo derecho sobre las bases de datos, cuando éstas contienen precisamente datos generados por maquinas. En este caso la finalidad última es la protección del mercado, la economía digital y la innovación basada en los datos.

\section{Conclusiones}

El valor económico de los datos en la sociedad actual es innegable, como también lo es el tráfico patrimonial de los mismos, tanto de los denominados raw data (los datos singulares provenientes directamente de un sujeto) como de los datos elaborados a través de técnicas de inteligencia artificial (big data) y los datos provenientes de maquinas. El fenómeno de mercantilización de los datos, que incluye tanto la contratación de bienes y servicios digitales, como el funcionamiento de la industria inteligente (industria 4.0) y el internet de las cosas (IoT) ha generado un amplio debate social sobre la participación de los particulares en el beneficio económico que los datos producen. En especial, desde un punto de vista jurídico, se ha puesto de manifiesto que es necesario regular de forma clara el poder de disposición y control que los sujetos tienen, o deben tener sobre los datos, en relación a su explotación patrimonial.

Partiendo de las premisas anteriores, la doctrina no coincide en la necesidad de reconocer que el poder de disposición de los sujetos de deba articular como un tipo de propiedad sobre los datos. Mientras algunos autores defienden que la institución de la propiedad es 
la fórmula adecuada para responder a esta necesidad de control, ya sea entendida como propiedad tradicional sobre bienes, o como propiedad intelectual, otros autores consideran que el poder de control se debe garantizar por otras vías.

Realmente, el reconocimiento de un derecho de propiedad sobre los datos se ha entendido más bien como una declaración de principios tendente tanto a obtener un control efectivo del sujeto sobre sus datos personales, como especialmente a participar en el rendimiento económico de todos los datos que genera, produciéndose una redistribución equitativa de los beneficios económicos de la economía de los datos. Sobre esta base, se ha planteado que tales objetivos se pueden obtener articulando un régimen contractual efectivo y protector del sujeto. Así, el desarrollo de normas de protección del consumidor resulta esencial.

En todo caso, una pretendida regulación debe tener en cuenta el principio de libre circulación de los datos. El respeto y defensa de este principio (declarado expresamente por la UE) se justifica por dos motivos esenciales: de un lado, la protección y defensa del libre mercado, principio esencial de funcionamiento de nuestra economía y base del crecimiento. Y, de otro lado, el interés público, ya que el tratamiento de datos está en la base de avances en materias tan importantes como la salud o la seguridad.

En suma, el desarrollo económico basado en el tratamiento de datos y la defensa de los derechos de los sujetos que generan los datos, incluyendo derechos patrimoniales, se deben compatibilizar, como ya señalaba el Supervisor Europeo de Protección de Datos en su Opinión 7/2015. Mientas la protección de los derechos unidos a la vertiente personal de la protección de la privacidad y la protección de datos personales se ha ido desarrollando y articulando de manera bastante satisfactoria en la UE, la regulación del aspecto patrimonial es una asignatura pendiente, la realidad, como siempre, va delante de la norma.

En relación con los datos personales debemos tener en cuenta que el reconocimiento de un derecho de propiedad puede no ser la vía más adecuada para proteger la privacidad y el derecho fundamental a la protección de los datos personales. Articular un sistema jurídico similar al modelo de los derechos de la personalidad, en especial la imagen, con una vertiente patrimonial reconocida y limitada, puede ser una fórmula a tener en cuenta. 
Junto a ello, la regulación a través de las normas contractuales es imprescindible, ya se ha iniciado el camino en la Unión Europea a través de las Directivas (UE) 2019/770 y 2019/771, que tienen que ser transpuestas y completadas por los Estados miembros. Las normas contractuales deben prestar especial atención a la protección del consumidor cuando la prestación a la que se compromete, o parte de ella, es el tratamiento de datos. Probablemente, como se ha propuesto en la doctrina, esta protección se debe extender a los casos en los que se tratan datos no personales o datos generados por maquinas, aunque se deban diferenciar las soluciones.

En relación con los datos no personales y, en especial, los datos industriales (en general, los generados por maquinas) parece existir un consenso doctrinal y también social en que no es necesario, ni acaso conveniente, el reconocimiento de un derecho de propiedad sobre ellos. En este ámbito resulta esencial la garantía de la libre circulación de los datos y el derecho de acceso. Junto a ello, la protección de la inversión tecnológica hecha por las empresas se podría articular, según proponen diversos autores, por la vía de un nuevo derecho de propiedad intelectual, cuyas reglas se deben conjugar con los principios de protección de datos personales y la protección del consumidor.

\section{Referencias bibliográficas}

CÁMARA LAPUENTE, S. (2021), "Un primer balance de las novedades del RDL 7/2021, de 27 de abril, para la defensa de los consumidores en el suministro de contenidos y servicios digitales (La transposición de las Directivas 2019/770 y 2019/771” (I). Diario La Ley n 9881. Sección Tribuna, 29 de junio de 2021, pp. 1-32.

DE FRANCESCHI, A. - SCHULZE, R. (2019), Digital revolution-New challenges for law. München/Baden-Baden, C.H. Beck/Nomos.

DREXL, J. (2018), "Data Access and control in the era of connected devices. Study on behalf of the European Consumer Organisation BEUC”. Bureau Européen des Unions des Consommateurs (BEUC). Brussels. https://www.beuc.eu/publications/beuc-X-2018-

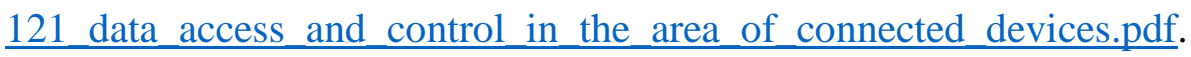


DREXL, J. (2019), "Legal challenges of the changing role of personal and non-personal data in the data economy" en: DE FRANCESCHI, A. - SCHULZE, R. (dirs.), Digital Revolution - New challenges for law. München/Baden-Baden, C.H. Beck/Nomos, pp. 1941.

DREXL, J. (2016), Designing Competitive Markets for Industrial Data - Between Propertisation and Access. Max Planck Institute for Innovation and Competition. Research Paper No. 16-13 (SSRN 2862975)

DUCH-BROWN, N. - MARTENS, B. - MUELLER-LANGER, F. (2017), The economics of ownership, access and trade in digital data. JRC Digital Economy Working Paper 2017-01.

FEZER, K-H (2017), "Data property for the people- An intrinsic intellectual property law sui generis regarding people`s behaviour-generated informational data". Zeitschrift für Geistiges Eigentum, 9, pp. 356-370.

FEZER, K-H (2018), Repräsentatives Dateneigentum Ein zivilgesellschaftliches Bürgerrecht. Estudio para la Fundación Konrad Adenauer. Accesible en https://www.kas.de/c/document_library/get_file?uuid=f828a351-a2f6-11c1-b720$\underline{1 \text { aa08eaccff9 \&groupId=252038. }}$.

GONZÁLEZ PACANOWSKA, I. (2020), “El derecho a la portabilidad de los datos personales: control y uso compartido de los datos personales" en GONZÁLEZ PACANOWSKA, I. (coord.), Protección de datos personales, Valencia, Tirant lo Blanch, pp. 668-731.

HUMMEL, P. - BRAUN, M. - DABROCK, P. (2020), “Own data? Ethical reflections on Data Ownership". Philosophy \& Technology. Accesible en https://doi.org/10.1007/s13347-020-00404-9.

JANECEK, V. - MALGIERI, G. (2020), “Commerce in data and the dynamically limited alienability rule". German Law Journal, 21, pp. 924-943. 
JANECEK, V. - MALGIERI, G. (2019), "Data extra commercium” en LOHSSE, S. SCHULZE, R. - STAUDENMAYER, D. (dirs.), Data as Counter-PerformanceContract Law 2.0?, Hart/Nomos. Baden-Baden. pp. 95-125. 
KERBER, W. (2016), “A New (Intellectual) Property Right for Non-Personal Data? An Economic Analysis'. Joint Discussion Paper Series in Economics No. 37-2016. Accesible en https://www.unimarburg.de/fb02/makro/forschung/magkspapers/paper_2016/372016_kerber.pdf

LANDREAU, I. - PELIKS, G. - BINCTIN, N. - PEZ-PÉRARD, V. (2018), Rapport “Mes data sont à moi. Pour une patrimonialité des données personnelles". Accesible en https://www.generationlibre.eu/wp-content/uploads/2018/01/2018-01generationlibrepatrimonialite-des-donnees.pdf.

NAVAS NAVARRO, S. (2018), "El valor de los datos personales en el mercado", en KINDL, J. - ARROYO VENDRELL, T. - GSELL, B. (Hrsg.), Verträge über digitale Inhalte und digitale Dienstleistungen, Baden-Baden, Nomos, pp. 101-122

OSBORNE CLARKE (2016), Legal study on ownership and access to data. A study prepared for the European Commission. Accesible: https://op.europa.eu/es/publicationdetail/-/publication/d0bec895-b603-11e6-9e3c-01aa75ed71a1

PERETE RAMIREZ, C. - GARCÍA MEXIA, P. (2021), "La propiedad sobre el dato ¿cabe una vertiente patrimonial de la protección de datos?” en TRONCOSO, A. (dir.), Comentario al Reglamento General de Protección de Datos y a la Ley Orgánica de Protección de Datos Personales y Garantía de los derechos Digitales, I, Cizur Menor. Aranzadi, pp. 1681-1700.

PLANA ARNALDOS, Ma C (2020), “Datos como contraprestación”, en GONZÁLEZ PACANOWSKA, I. (coord.), Protección de datos personales, Valencia, Tirant lo Blanch, pp. 561-618.

PRAINSACK, B. (2019), "Logged out: Ownership, exclusion and public value in the digital data and information commons". Big Data \& Society. 1-15. DOI: $10.1177 / 2053951719829773$. 
PURTOVA, N. (2010), "Property in Personal Data: a European Perspective on the Instrumentalist Theory of Propertisation", European Journal of Legal Studies, 2, 3, pp. 193-208. Accesible en http://hdl.handle.net/1814/15124.

PURTOVA, N. (2015), “The Illusion of Personal Data as No One's Property". Law, Innovation, and Technology, 7, pp. 83-11

RITTER, J. - MAYER, A., "Regulating data as property: a new construct for moving forward", Duke Law and Technology Review, 16, pp. 220-277.

THOUVENIN, F. - TAMÓ-LARRIEUX, A. (2021), "Data Ownership and Data Access Rights." en Big Data and Global Trade Law, Cambridge University Press, pp. 316-339. DOI: https://doi.org/10.1017/9781108919234.020

VICTOR, J.M (2013), “The EU General Data Protection Regulation. Toward a property regime for protecting data privacy", The Yale Law Journal, 123, pp. 513-528.

ZECH, H. (2016), "Data as a Tradeable Commodity”, en DE FRANCESCHI, A. (ed.), European Contract Law and the Digital Single Market: The Implications of the Digital Revolution, Cambridge, Intersentia, pp. 51-80.

ZECH, H. (2017), "Data as a tradable commodity. Implications for contract Law", DREXL, J. (ed.), Proceedings of the 18th EIPIN Congress: The New Data Economy between Data Ownership, Privacy and Safeguarding Competition. Accesible en: SSRNid3063153.pdf. 